\title{
Serum-Derived Bovine Immunoglobulin/ Protein Isolate Therapy for Patients with Refractory Irritable Bowel Syndrome
}

\author{
Leonard B. Weinstock ${ }^{1}$, Victoria S. Jasion² \\ ${ }^{1}$ Specialists in Gastroenterology, St. Louis, USA \\ ${ }^{2}$ Department of Medical Affairs, Entera Health, Inc., Cary, USA \\ Email: Iw@gidoctor.net
}

Received 10 August 2014; revised 25 September 2014; accepted 12 October 2014

Copyright (C) 2014 by authors and Scientific Research Publishing Inc.

This work is licensed under the Creative Commons Attribution International License (CC BY). http://creativecommons.org/licenses/by/4.0/

cC) (i) Open Access

\begin{abstract}
Background: A small double-blind study showed benefits of serum-derived bovine immunoglobulin/protein isolate (SBI), for diarrhea-predominant irritable bowel syndrome (IBS-D) [1]. The purpose of this chart review was to assess safety and clinical outcomes of SBI in refractory irritable bowel syndrome (IBS) patients. Methods: A retrospective review of 35 IBS patients with diarrhea or mixed diarrhea/constipation pattern (IBS-M) who were administered SBI 5 grams twice daily was performed. Clinical response ("good response" or "no response") and adverse events were determined by follow-up after four weeks of therapy. Patients were included for evaluation if a lactulose breath test (LBT) had been performed prior to SBI. All patients were refractory to common IBS therapies. The response rate to the inclusion of SBI was calculated in three separate groups: dividing patients based on their LBT results (positive or negative), dividing patients by their IBS diagnosis (IBS-D or IBS-M) and grouping all patients together. Results: Analysis was carried out on 26 IBS-D/-M patients with LBT results. Two patients were lost to follow-up and were excluded from data analysis. The positive LBT group $(N=11)$ had a $73 \%(p=0.117)$ positive response rate to SBI. The negative LBT group $(\mathrm{N}=13)$ had a significant response rate of $77 \%(p=$ 0.040). If patients were divided by IBS diagnosis or grouped together, the response rate to SBI was similarly ranging from 69\% - 88\%. Adverse events leading to cessation of SBI occurred in 3 of 24 patients. Conclusion: SBI appeared to be a safe and effective nutritional moiety in refractory IBS-D and IBS- M patients. Larger, double-blind studies are needed.
\end{abstract}

\section{Keywords}

Irritable Bowel Syndrome (IBS), Small Intestinal Bacterial Overgrowth (SIB0), Serum-Derived Bovine Immunoglobulin/Protein Isolate (SBI) 


\section{Introduction}

Irritable bowel syndrome (IBS) is a common gastrointestinal disorder affecting $10 \%-15 \%$ of adults [2]. An estimated $28 \%$ of visits to gastroenterologists and $12 \%$ of visits to primary care offices are from patients with IBS [3]. The pathophysiology of IBS is complex. Recently, gastrointestinal inflammation and altered gut microflora have been implicated [4] [5].

Therapeutic options are diverse and include dietary modification, fiber supplementation, psychological therapy (counseling, hypnosis, relaxation, etc.) and pharmacological therapy (prescription drugs, over-the-counter medications, herbs and dietary supplements) [6]. The number of FDA-approved medications indicated for IBS is limited. New algorithms for IBS therapy based on the role of bacterial overgrowth, in particular small-intestinal bacterial overgrowth (SIBO), have been proposed [7].

Serum-derived bovine immunoglobulin/protein isolate (SBI), a FDA-regulated medical food product, is composed of greater than $50 \%$ immunoglobulin and has been shown to survive the stomach environment and bind microbial components in the intestine, thereby neutralizing their effects in many different animal models [8]. In a double-blind, randomized, placebo-controlled IBS-diarrhea (IBS-D) study, 10 grams of SBI per day were shown to cause a statistically significant decrease the number of days per week that patients experience-abdominal pain, flatulence, urgency, loose stools, bloating or any symptom [1]. The microbial binding activity of SBI may have downstream effects in maintaining GI immune balance and managing gut barrier function, which ultimately leads to improved nutrient utilization [8]. In addition to the gut microflora being a key player in the complex pathophysiology of IBS, it has recently been shown that IBS-D patients have abnormal tight junction proteins in the jejunal mucosa versus healthy adults [9]. Thus, both the endotoxin binding and barrier function maintenance properties of SBI are particularly relevant in this patient population for nutritional management of their conditions.

The purpose of this retrospective chart review was to assess IBS-patient response, within a community gastroenterology practice, to the inclusion of a nutritional therapy, SBI, into their diet as part of their standardof-care. This chart review summarizes the response to 5 grams SBI twice per day (BID) administered over four weeks in patients who were first screened for SIBO using the lactulose breath test (LBT).

\section{Methods and Materials}

\subsection{Patient Population}

Initial IBS diagnosis of all patients in this chart review was based upon patient-reported symptoms in a private gastroenterology practice. All patients were diagnosed by the same physician (LBW) between April 2014 and July 2014 using the Rome II criteria [10]. The initial patient questionnaire asks the patient to identify bowel habit: "no problems", "mainly constipation", "alternating diarrhea and constipation" and "mainly diarrhea". The patient form also has a six-point scale (0—not at all, 6-a very great deal) assessing how bothersome the following symptoms are to patients: abdominal discomfort, abdominal bloating and flatulence/lower gas. Finally, patients were asked whether or not the discomfort had occurred at least 3 months per year. If a patient was diagnosed with either diarrhea-predominant IBS (IBS-D) or mixed diarrhea/constipation pattern (IBS-M) from this questionnaire, a lactulose breath test (LBT) was performed to screen for SIBO. At a routine four-week follow-up, the patients were seen in the office and when this did not occur, the office nurse called patients to assess progress. Since the follow-up did not consist of the same questionnaire, this data is not reported. Only patients who had the LBT performed prior to adding SBI to their diets were included in this review.

All LBT were performed using the QUINTRON Breath Tracker HC. A positive lactulose hydrogen breath test (LBT) is considered $20 \mathrm{ppm}$ over the basal amount of breath hydrogen, at or before 90 min of ingesting lactulose. Methane excretion of $3 \mathrm{ppm}$ or more was determined to be clinically significant and two antibiotics were administered if hydrogen was abnormal and methane was present.

\subsection{Data Extraction and Analysis}

The criteria for this chart review were diagnosis of IBS with either diarrhea or mixed diarrhea/constipation pattern (IBS-D or IBS-M) according to Rome II criteria, pre-therapeutic results from LBT and patients ingesting 5 grams SBI BID for four weeks. Of 35 potential IBS patients, 26 patients fulfilled those criteria and were divided into two groups: patients who either had a positive LBT (+LBT) or those with negative LBT (-LBT) prior to any 
therapy. Before being placed on SBI, all patients with a positive LBT were first placed on a 14-day course of 550 $\mathrm{mg}$ rifaximin three times per day (TID) with or without metronidazole or neomycin. Many of these patients were then placed on a variety of other common IBS treatments, including tricyclic anti-depressants, loperamide, anticholinergics and diet modification (FODMAP). If there was an incomplete response after these therapies, then they were prescribed SBI as a nutritional standard-of-care. All patients who initially had a negative LBT and who had failed conventional IBS treatments to sufficiently manage their condition as determined by the clinician (FODMAP diet, probiotics, tricyclic anti-depressants and/or alosetron) were placed on 5 grams SBI BID for four weeks without other therapies. From each group, one patient was lost to follow-up. For data analysis, these patients were not included in the total sample size or analysis. The primary goal of this report is to assess patient satisfaction and response to SBI in a community gastroenterology practice, so patients were categorized as either "good response" or "no response" within their LBT groups. This outcome was asked and recorded in patient's chart as standard-of-care in the clinician's private practice (LBW-Specialists in Gastroenterology, St. Louis, MO., United States) at a four-week follow-up interval.

The previous clinical trial of SBI was in IBS-D [1], however, IBS-M patients were included in this chart review. Therefore, after patients were grouped and analyzed according to their LBT outcome (positive or negative), patients in this chart review were pooled again and re-grouped as either IBS-D patients or IBS-M patients, irrespective of the LBT results. The clinical response rate was then calculated in each of these populations regardless of their LBT results. Finally, all patients were grouped together and the broad response rate was calculated.

Overall, the percentage of patients who responded to SBI was calculated within either their LBT group, their IBS category (IBS-D or IBS-M) or the entire patient group. A one-sample t-test between the percentages of "good response" versus "no response" within both groups was used to determine if patient outcomes were significant. Statistics calculations were performed with the StatPac statistics calculator [11].

\section{Results}

Twenty four patients were included in this retrospective chart review. Table 1 outlines the patients as organized by the LBT outcomes, either positive or negative. In the positive LBT group, the number of patients who had failed common therapies was as follows: rifaximin (10); low dose naltrexone (4); probiotics (4); tricyclic antidepressants (3); neomycin (3); metronidazole (3); ampicillin (1); loperamide (1); linaclotide (1); FODMAP diet (1). In the negative LBT group, the number of patients who had failed common therapies was as follows: rifaximin (8); low dose naltrexone (4); probiotics (4); neomycin (4); tricyclic antidepressants (3); linaclotide (2); gluten-free diet (2); metronidazole (1); trimethoprim/sulfamethoxazole (1); SSRI (1); cromalyn (1); aloestron (1); colestipol (1); bismuth (1).

The percentage of patients who responded positively to the inclusion of SBI into their diets was high for all patient groups. The positive LBT group $(\mathrm{N}=11)$ had a response rate of $73 \%, t(10)=1.718, p=0.117$ and the negative LBT group $(\mathrm{N}=13$ ) had a significant response rate of $77 \%, t(12)=2.31, p=0.040$ (Table 1$)$. If patients were separated by their diagnosis, a similar high-response rate was noted. IBS-D patients $(\mathrm{N}=16)$ had a $69 \%$ response rate, $t(15)=1.64, p=0.121$ and IBS-M patients $(\mathrm{N}=8)$ had a significant response rate of $88 \%, t$ $(7)=3.2, p=0.015$. When all patients were pooled $(\mathrm{N}=24)$, the response rate of $75 \%$ was significant with $t$ $(23)=2.83, p=0.010$.

The adverse events (AEs) led to cessation of therapy in 3 of 24 patients and included: 1 patient who reported constipation, 1 patient who reported diarrhea and 1 patient who reported nausea and constipation. These adverse events were short-lived and self-limited.

\section{Discussion}

The data presented show positive clinical response among refractory IBS-D/-M patients in a community gastroenterology practice to the inclusion of 5 grams SBI twice per day (BID) over four weeks as a nutritional therapy administered as standard-of-care. Whether patients were divided by LBT outcome, initial IBS diagnosis, or pooled together, the response rate was between $69 \%-88 \%$. The LBT group was composed of two groups: either IBS-D/-M patients with positive LBT who were refractory to rifaximin therapy and other IBS therapies with an SBI response rate of $73 \%(p=0.117)$, or IBS-D/-M patients with negative LBT who were also refractory to common IBS therapies with a significant response rate of $77 \%(p=0.040)$. Although the former group did not obtain statistical significance, both groups had a similar response rate. When patients were divided by diagnosis, 
Table 1. Clinical response from IBS-D/IBS-M patients with LBT results ingesting 5 grams SBI twice daily for four weeks.

\begin{tabular}{|c|c|c|}
\hline & \multicolumn{2}{|c|}{ Group } \\
\hline & IBS-D ${ }^{\mathrm{a} / I B S}-\mathrm{M}^{\mathrm{b}}$ with positive $\mathrm{LBT}^{\mathrm{c}} \neq$ & IBS-D/IBS-M with negative LBT \\
\hline Total Number $\left(\#^{\mathrm{d}}\right)$ of Patients & 11 & 13 \\
\hline Percent Reseponse & $73 \%, p=0.117$ & $77 \%, p=0.040$ \\
\hline \# IBS-D & 7 & 9 \\
\hline \# IBS-M & 4 & 4 \\
\hline \# Male & 3 & 5 \\
\hline \# Female & 8 & 8 \\
\hline Average Age & $48 \pm 16$ & $47 \pm 18$ \\
\hline \# Responded to $\mathrm{SBI}^{\mathrm{e}}$ & 8 & 11 \\
\hline \# Without Response to SBI & 3 & 3 \\
\hline \# Adverse Events & 1 & 2 \\
\hline Reported Adverse Events & constipation & diarrhea, nausea and constipation \\
\hline
\end{tabular}

$\ddagger$ Before being administered SBI, all patients had reported an incomplete clinical response to a 14-day course of rifaximin, $550 \mathrm{mg}$ three times per day (TID); ${ }^{2}$ Diarrhea-predominant irritable bowel syndrome (IBS-D); ${ }^{\mathrm{b}}$ Mixed diarrhea/constipation pattern irritable bowel syndrome (IBS-M); ${ }^{c}$ Lactulose breath test (LBT); ${ }^{\mathrm{d}}$ Number (\#); ${ }^{\mathrm{e}}$ Serum-derived bovine immunoglobulin/protein isolate (SBI).

IBS-D patients had a $69 \%$ response rate $(p=0.121)$ and IBS-M patients had a significant response rate of $88 \%$ $(p=0.015)$. This suggested that SBI might be useful for IBS-M, yet the clinical trial of SBI was only conducted in IBS-D populations [1]. When pooled together, the clinical response rate to the inclusion of SBI as a nutritional therapeutic for these intractable IBS-D/-M patients was statistically significant at 75\% $(p=0.010)$. The statistical variation observed in this small case analysis between groups suggests a need for a larger and well-powered study to examine this particular population, the LBT positive group of IBS-D and IBS-M subjects.

This chart review suggests the utility of SBI in refractory IBS-D/-M patients who had negative or positive LBT. The patients with a negative LBT had also failed previous therapies and had a statistically significant response rate to SBI. The reason why patients are grouped into their pre-therapeutic LBT outcomes is that this is the only objective measurement a clinician can use in IBS patients. This chart review is also the first formal report of a significant response rate to SBI in IBS-M patients. However, by regrouping patients into their IBS diagnosis (IBS-D or IBS-M) and disregarding their LBTs, the outcomes lack any objective variables. The same can be said regarding the statistically significant response rate of $75 \%$ when all patients were grouped together. Nonetheless, all patients had intractable IBS-D/-M, illustrating that SBI could be useful in refractory patients. This retrospective chart review presents general clinical data that is supporting of the finding of the doubleblind study of SBI in IBS-D, which illustrates improvements in the number of days per week that patients reported loose stools, abdominal pain, flatulence, urgency and bloating [1]. The low number of patients who reported adverse events while ingesting SBI was such that 21 of 24 patients were able to complete 4 weeks of therapy.

There are several caveats to this chart review. This is a retrospective analysis so results were based upon standard-of-care patient outcomes utilizing SBI for nutritional management without a control group. In addition, there were no follow-up LBTs after recording a clinical response to SBI. The patient-reported improvement is limited to a simple "good response" or "no response", meaning there is no detailed gauge for clinical outcome. This is also a relatively small chart review, with only 26 patients fulfilling the inclusion criteria and 24 patients with complete follow-ups. Given the inclusion criteria of this retrospective review, there are groups which are not included in this study, so it is difficult to make broader conclusions outside of those already mentioned. Specifically, there is no group of IBS (IBS-D or IBS-M) patients with a positive LBT who declined rifaximin and were immediately placed on SBI for nutritional management of their conditions. In addition, there is no group of IBS-D/-M patients without any LBT testing who were placed on SBI, though current clinical results seem to al- 
ready support this use [1]. Finally, it is impossible to make conclusions which directly compare the clinical response rate between + LBT and -LBT groups reported in this review since there are factors which are inherently confounding. All patients in the +LBT were first treated with antibiotic therapy and had failed this therapy.

As aforementioned, the purpose of chart review is to analyze the patient response to SBI and to report the in-office, open-label clinical observation. The data suggest that despite the presence or absence of SIBO, SBI appears to have a similar effect in IBS-D/IBS-M patients who are refractory to common IBS treatments. In animal models SBI has been beneficial in reducing inflammation and binding bacterial toxins [8] [12]. Oral immunoglobulins in animals have been shown to neutralize bacterial infection and reduce inflammation [13]. In a HIV positive population with enteropathy, SBI was shown to decrease proinflammatory Gammaproteobacteria and decrease Clostridium (genus) over a period of eight weeks [14]. Other in vitro results have demonstrated that SBI binds to and neutralizes $C$. difficile toxins A and B [15].

Further controlled clinical studies are needed to elucidate the utility of SBI for therapy of IBS patients diagnosed with and without SIBO and in IBS-M populations. Based on its mechanism of action, SBI may have broad potential for use in the management of several different infective and non-infective enteropathies potentially in combination with antibiotics or as part of dietary management protocol in these patients.

\section{Acknowledgements}

Authors would like to thank Entera Health for their careful review and editing of this manuscript.

\section{References}

[1] Wilson, D., Evans, M., Weaver, E., Shaw, A.L. and Klein, G.L. (2013) Evaluation of Serum-Derived Bovine Immunoglobulin Protein Isolate in Subjects with Diarrhea-Predominant Irritable Bowel Syndrome. Clinical Medicine Insights: Gastroenterology, 6, 49-60. http://dx.doi.org/10.4137/CGast.S13200

[2] Saito, Y.A., Schoenfeld, P. and Locke, G.R. (2002) The Epidemiology of Irritable Bowel Syndrome in North America: A Systematic Review. The American Journal of Gastroenterology, 97, 1910-1915. http://dx.doi.org/10.1016/S0002-9270(02)04270-3

[3] Ringel, Y. and Drossman, D.A. (2000) Toward a Positive and Comprehensive Diagnosis of Irritable Bowel Syndrome. Medscape General Medicine 2.

[4] Collins, S.M. (2014) A Role for the Gut Microbiota in IBS. Nature Reviews Gastroenterology \& Hepatology, 11, $497-$ 505. http://dx.doi.org/10.1038/nrgastro.2014.40

[5] Simren, M., Barbara, G., Flint, H.J., Spiegel, B.M., Spiller, R.C., Vanner, S., Verdu, E.F., Whorwell, P.J., Zoetendal, E.G. and Rome Foundation, C. (2013) Intestinal Microbiota in Functional Bowel Disorders: A Rome Foundation Report. Gut, 62, 159-176. http://dx.doi.org/10.1136/gutjnl-2012-302167

[6] Enck, P., Junne, F., Klosterhalfen, S., Zipfel, S. and Martens, U. (2010) Therapy Options in Irritable Bowel Syndrome. European Journal of Gastroenterology \& Hepatology, 22, 1402-1411. http://dx.doi.org/10.1097/MEG.0b013e3283405a17

[7] Pimentel, M. (2010) An Evidence-Based Treatment Algorithm for IBS Based on a Bacterial/SIBO Hypothesis: Part 2. The American Journal of Gastroenterology, 105, 1227-1230. http://dx.doi.org/10.1038/ajg.2010.125

[8] Petschow, B.W., Burnett, B., Shaw, A.L., Weaver, E.M. and Klein, G. L. (2014) Serum-Derived Bovine Immunoglobulin/Protein Isolate: Postulated Mechanism of Action for Management of Enteropathy. Clinical and Experimental Gastroenterology, 7, 181-190. http://dx.doi.org/10.2147/CEG.S62823

[9] Martinez, C., Lobo, B., Pigrau, M., Ramos, L., Gonzalez-Castro, A.M., Alonso, C., Guilarte, M., Guila, M., de Torres, I., Azpiroz, F., Santos, J. and Vicario, M. (2013) Diarrhoea-Predominant Irritable Bowel Syndrome: An Organic Disorder with Structural Abnormalities in the Jejunal Epithelial Barrier. Gut, 62, 1160-1168. http://dx.doi.org/10.1136/gutjnl-2012-302093

[10] Thompson, W.G., Longstreth, G.F., Drossman, D.A., Heaton, K.W., Irvine, E.J. and Muller-Lissner, S.A. (1999) Functional Bowel Disorders and Functional Abdominal Pain. Gut, 45, II43-II47. http://dx.doi.org/10.1136/gut.45.2008.ii43

[11] Walonick, D.S. (1996-2014) StatPac Statistics Calculator.

[12] Petschow, B.W., Burnett, B.P., Shaw, A.L., Weaver, E.M. and Klein, G.L. (2014) Dietary Requirement for Serum-Derived Bovine Immunoglobulins in the Clinical Management of Patients with Enteropathy. Digestive Diseases and Sciences, in press. http://dx.doi.org/10.1007/s10620-014-3322-0

[13] Bosi, P., Casini, L., Finamore, A., Cremokolini, C., Merialdi, G., Trevisi, P., Nobili, F. and Mengheri, E. (2004) 
Spray-Dried Plasma Improves Growth Performance and Reduces Inflammatory Status of Weaned Pigs Challenged with Enterotoxigenic Escherichia Coli K88. Journal of Animal Science, 82, 1764-1772.

[14] Asmuth, D.M., Strombaugh, J., Ursell, L., Ma, Z.M., Albanese, A., Hodzic, E., Troia-Canico, P., Flynn, N.M., Yotter, T., Miller, C.J. and Knight, R. (2013) Changes in Stool Microbiota, Bacterial Translocation and Mucosal Immunity after Oral Serum-Derived Bovine Immunoglobulin (SBI) Administration. 20th Conference on Retroviruses and Opportunistic Infections (CROI), Atlanta, 3-6 March 2013.

[15] Henderson, A.L., Horgan, A., Detzel, C.J., Maas, K.J. and Weaver, E. (2014) Serum-Derived Bovine Immunoglobulin/Protein Isolate Binds and Neutralizes Clostridium Difficile Toxins A and B. Digestive Disease Week (DDW), Chicago, 3-6 May 2014. 
Scientific Research Publishing (SCIRP) is one of the largest Open Access journal publishers. It is currently publishing more than 200 open access, online, peer-reviewed journals covering a wide range of academic disciplines. SCIRP serves the worldwide academic communities and contributes to the progress and application of science with its publication.

Other selected journals from SCIRP are listed as below. Submit your manuscript to us via either submit@scirp.org or Online Submission Portal.
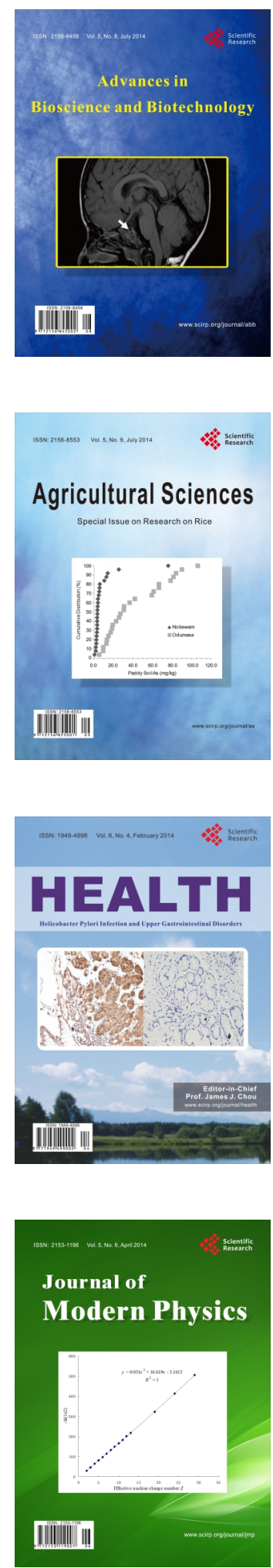
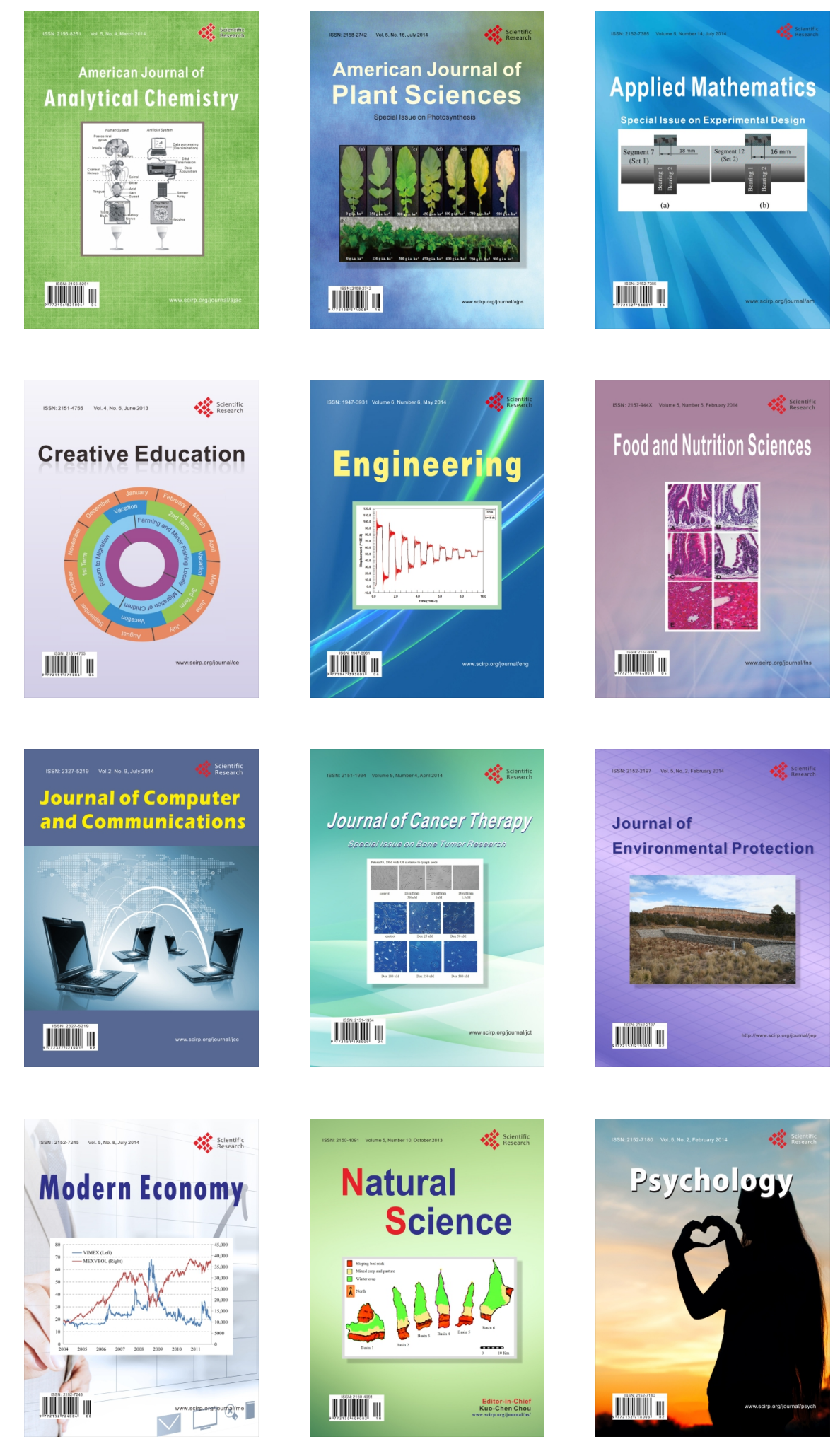